\title{
Désignation et classement des sols agricoles d'après des critères de situation et d'organisation : application aux terres franc-comtoises du domaine climatique tempéré semi-continental
}

\author{
S. Bruckert \\ Pédologie, Université de Franche-Comté, place Leclerc, 25030 Besançon, France \\ (reçu le 1-6-1988, accepté le 5-1-1989)
}

Résumé - La méthode de désignation des sols proposée s'appuie sur la description et l'analyse de très nombreux profils, prospectés sur environ 40000 ha des plateaux calcaires et marno-calcaires francs-comtois de basse altitude. L'auteur en expose le principe et développe les éléments qui permettent, même à des non-spécialistes en pédologie, de l'appliquer et d'en tirer profit pour acquérir une connaissance des constituants, des propriétés essentielles et des contraintes des terres agricoles régionales.

Le sigle de désignation des sols trouve sa première application dans la clé dichotomique mise au point pour ranger les sols agricoles dans l'une des 6 classes d'aptitude à une mise en culture polyvalente (blé, maïs, colza, prairie). Elle met en jeu l'interaction climat-sol pour définir une profondeur "rhizofonctionnelle" optimum, ainsi que la pente, l'aération des horizons profonds, l'obstacle à l'enracinement, la texture et la pierrosité. Les critères retenus soulignent l'importance des caractères physiques dans les potentialités des sols et dans les relations sols-plantes. La méthode proposée peut être facilement appliquée hors du contexte régional. C'est un outil de synthèse, utilisable en cartographie des sols à but finalisé.

relations sols-plantes - interaction climat-sol - profondeur d'enracinement - contrainte physique du sol - aptitude à la mise en culture

Summary - Designation and classification of farming soils according to site and organization criteria: application to franc-comtois lands of semi-continental temperate climatic zone. The proposed method of the soil designation relies on the description and analysis of numerous soils, propected over about 40000 ha of the francs-comtois calcareous and marly-calcareous plateaus at low altitude. The author defines the principe and develops the factors allowing, even to non-pedologists, to apply it and to deliver a knowledge of the components, and of the essential properties and limitations of agricultural and regional lands.

The acronym of the soil designation has its first application in a dichotomous key created to place the main soils series into one of 6 "ease of cultivation" classes. This includes the interaction climate-soil to define the maximum depth in correlation with that of roots. Criteria considered to have an important effect on cultivation and yield are available moisture, slope, air capacity of subsoil horizons, depth of penetration by plant roots, nature of obstacles preventing root penetration, particle-size class and stoniness. These properties show the great importance of the soil physical conditions to classify land for agriculture according to its potential and the severity of its limitations for crop growth. These criteria may prove suitable for categorizing other soils outside the region. The land classification proposed is suitable for describing soils in the survey areas.

plant-soil relations - climate-soil interaction - root penetration depth - soil physical limitations - ease of cultivation

\section{Introduction}

De nombreux travaux portent sur la classification des sols en fonction de leurs potentialités et de leurs contraintes vis-à-vis de la croissance des plantes cultivées (Jamagne et al., 1966; Lajoie, 1975; Begon et al., 1978; Commission canadienne de pédologie, 1978; Mori et al., 1983). Les progrès réalisés dans ce domaine ont fait l'objet, en 1983, d'une confrontation menée à l'échelle des états membres de la Communauté européenne (Haans et al., 1984). La plupart des démarches adoptées visent à estimer l'aptitude des sols à l'égard d'une culture donnée, telles les études de Harrod (1979) et de Hallaire (1981) sur 
les sols de prairie. En revanche, peu de travaux ont pour objectif d'apprécier l'aptitude à la mise en culture considérée sous un angle non restrictif (Jamagne et al., 1966; Begon et al., 1978; Mori et al., 1983). Bibby et Mackney (1969), en Grande-Bretagne, ont établi une classification des sols en fonction de leur land use capability. Les travaux de Thomasson (1971) et de Jones (1975 et 1979) ont suivi la même démarche. Duclos (1971) a adopté une méthodologie très différente en attribuant à chaque critère de classement un poids chiffré, la somme de ces notes donnant une valeur au sol. Les praticiens suisses (Guide pour l'estimation de domaines et de bien fonds agricoles, 1979) utilisent également un classement chiffré des sols, mais en régionalisant climatiquement leur estimation. Ces deux dernières méthodes, dont la mise en œuvre reste difficile, ont l'inconvénient de ramener le sol à un chiffre et de ne pas expliciter le classement par les critères exprimés en clair. L'emploi d'une clé dichotomique, introduisant successivement les critères, présente l'avantage d'indiquer l'itinéraire de classement suivi (Mondon, 1978). C'est dans cette direction que nous avons orienté nos recherches.

Les méthodes de désignation et de classement des sols agricoles que nous présentons ont été mises au point de 1982 à 1984, au cours de plusieurs séries de campagnes de prospection pédologique sur les plateaux de Haute-Saône (Bruckert, 1986b), puis testées sur l'ensemble des plateaux francs-comtois de basse altitude, situés au nord-ouest des premiers plateaux jurassiens et au sud des collines sous-vosgiennes.

\section{Démarche et méthodologie}

Le choix des secteurs s'est opéré en accord avec les responsables de la Chambre régionale d'agriculture, dans le cadre de la Relance agronomique (Fig. 1). Au total, 40000 ha de sols cultivés ont été prospectés dans le but:

- d'observer sur le terrain les critères pédologiques et le comportement de l'ensemble terre arable-plantes face aux actions climatiques et humaines;

- de connaître les conditions d'environnement imposées aux sols et aux cultures;

- de définir les caractères pédologiques observables au champ pour renseigner sur le volume de sol prospecté par les racines;

- d'exprimer la composition et les contraintes des sols par une formule abrégée immédiatement perceptible par tous.

Nous avons complété cet acquis par des informations recueillies auprès des conseillers agricoles et des agriculteurs, au cours de plusieurs réunions où les concepts et les objectifs de chacun étaient exposés.
C'est ainsi que se sont peu à peu dégagés les éléments de deux outils:

- le sigle de désignation des sols (Bruckert, 1986a), qui indique de façon analytique les caractéristiques les plus essentielles des sols et de leur comportement, dans un langage accessible à tous ceux qui ne sont pas des spécialistes de science du sol, mais qui touchent de près ou de loin à la terre: agronomes, agriculteurs, géologues-conseils, ingénieurs de bureau d'étude...

- une clé de classement d'emploi simple, fondée sur la profondeur utile, ou profondeur "rhizofonctionnelle", effectivement prospectée par les plantes et sur des critères déterminés avec le plus d'objectivité possible (Bruckert, 1987). En effet, dans une même parcelle de terre, on observe couramment dans les rendements des différences significatives, liées aux propriétés des couches profondes. La croissance de la plupart des plantes cultivées dépend des possibilités offertes au système racinaire de se développer à la fois dans la couche labourée et dans les horizons sous-jacents d'un sol (Henin, 1960; Callot et al., 1983; Callot, 1984). L'alimentation en eau par les réserves situées sous la terre arable, permet de valoriser la fertilisation (Sebillotte et al., 1978). La colonisation racinaire de toutes les couches du sol est une condition nécessaire de prélèvement optimum des réserves en éléments nutritifs (Boiffin et al., 1982).

Le terme "rhizofonctionnel" que nous proposons nous paraît plus explicite et plus précis que le terme "utile" employé par les agronomes ou "physiologique" adopté par les praticiens suisses (Guide pour l'estimation de domaines et de bien-fonds agricoles, 1979).

\section{Principe et modalités d'expression du sigle de désignation des sols}

\section{Principe}

Le sol est désigné par une formule, ou sigle, sous forme de fraction qui comporte trois parties:

- au numérateur, la composition des constituants prospectés par les racines;

- au dénominateur, la composition des constituants qui font obstacle aux racines, avec indication de leur profondeur;

- latéralement, l'indication des contraintes qui amoindrissent les potentialités du sol.

\section{Détermination des constituants prospectés par les racines}

On observe que trois critères typiquement pédologiques favorisent la progression "mécanique" des racines et leur alimentation en air et en eau: ce sont les caractères aéré, poreux et meuble. Le premier est facilement apprécié par l'absence de signes d'oxydoréduction, le fer étant un indicateur coloré très sensible aux variations de teneur en oxygène; les deux autres sont évalués par la structuration, la friabilité, la facilité de pénétration à la tarière, la présence de terre arable enfouie en profondeur par la faune.

A partir des descriptions de terrain, on formule le numérateur du sigle en désignant, par leurs constituants texturaux, la terre arable et les horizons sousjacents prospectés par les racines (Tableau I). 


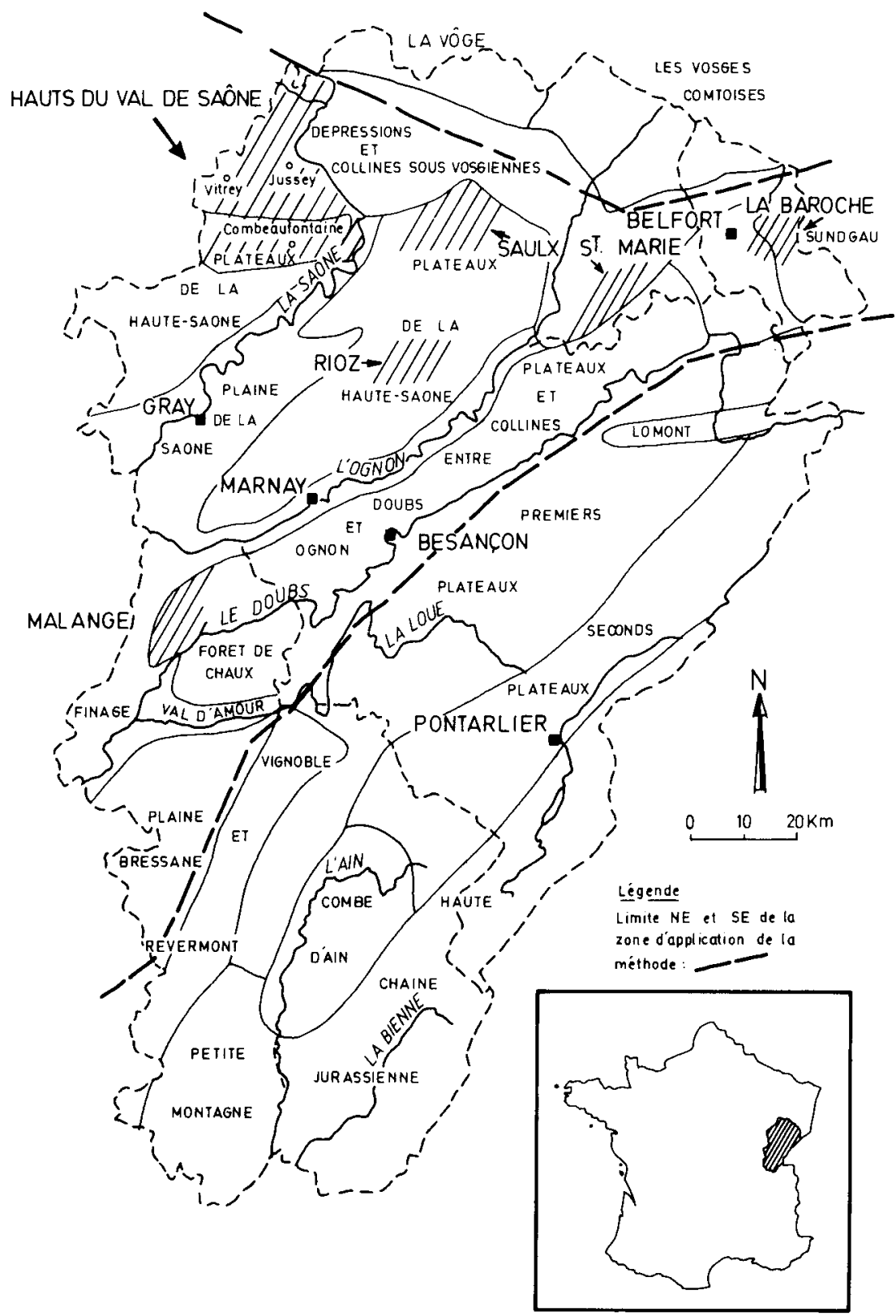

Fig. 1. Situation géographique des zones étudiées dans les régions naturelles franc-comtoises. (Fond cartographique des Régions Naturelles, Mathieu, 1976).

\section{Détermination de l'obstacle à l'enracinement}

Les obstacles qui limitent le développement des racines de la plupart des plantes cultivées sont soit physiques, soit chimiques. Les racines rencontrent divers obstacles physiques, dus au milieu naturel ou à des processus pédologiques. Nous les désignons par des lettres majuscules et leur profondeur d'apparition est notée en indice de 1 à 4 (Tableau I).

Un déficit en oxygène empêche le développement normal des racines de la plupart des plantes et limite, par-là, la profondeur d'enracinement (Russel, 1949; Grable et Siemer, 1968). II se manifeste dans le profil par des taches bleutées, verdâtres et blanchâtres, liées à la réduction du fer. Ces teintes peuvent colorer entièrement un horizon comme celui des gleys réduits, mais le plus souvent, elles sont associées à des taches rouille (hydroxydes ferriques) et à des précipi- tés noirâtres d'oxydes de fer et de manganèse. Elle se répartissent alors par plages juxtaposées sur le fond matriciel terne.

Contrairement aux obstacles physiques, la profondeur de l'obstacle induit par le manque d'oxygène est difficile à déterminer, car les signes d'oxydo-réduction peuvent se situer aussi bien dans les horizons renfermant des racines que dans les horizons sous-jacents. Pour surmonter la difficulté nous avons défini deux paramètres: l'un qui donne la profondeur d'apparition des taches d'oxydo-réduction (y), l'autre qui évalue la contrainte "manque d'oxygène" (g).

Le paramètre " $y$ " a l'avantage d'être objectif. Etant corrélé à la profondeur notée en indice de 1 à 4 , nous le plaçons au dénominateur du sigle (Tableau I). Le paramètre " $g$ " rend compte de l'intensité de l'anoxie. Comme son estimation est subjective, nous avons cherché à le définir avec plus de précision par un 
Tableau I. Sigle de désignation des sols: abréviations employées régionalement et application à un exemple commenté dans le texte.

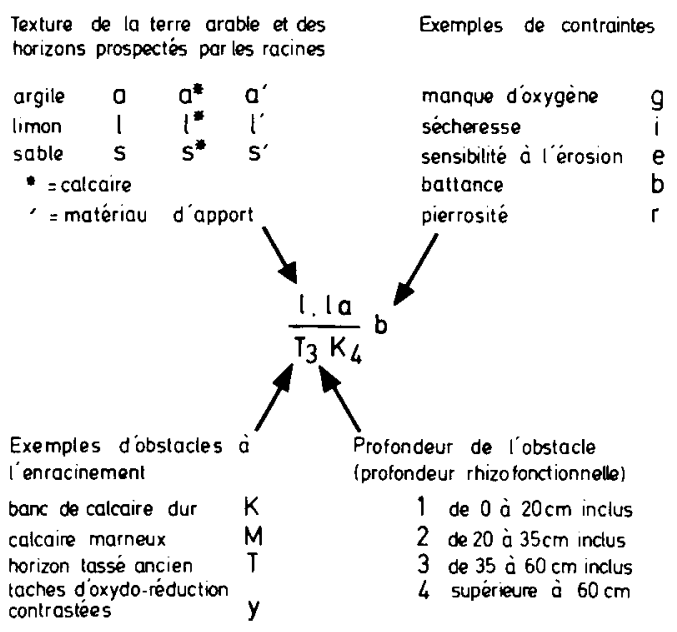

ensemble de critères d'observation portant sur l'enracinement, sur la structuration de la terre arable et sur la profondeur de l'horizon de pseudogley ou de gley. Nous distinguons 4 cas:

- $g_{1}$ ou indice d'une contrainte très forte, avec un enracinement limité à moins de $20 \mathrm{~cm}$, un horizon gleyifié à $20 / 25 \mathrm{~cm}$ et une structure détruite dans la terre arable même en été;

- $\mathrm{g}_{2}$ ou indice d'une contrainte forte, avec un enracinement limité à moins de $35 \mathrm{~cm}$, un horizon gleyifié à $25 / 40 \mathrm{~cm}$ et une terre arable structurée au moins en été;

- $g_{3}$ ou indice d'une contrainte moyenne, avec un enracinement limité à moins de $60 \mathrm{~cm}$, un horizon gleyifié à $40 / 60 \mathrm{~cm}$ et une terre arable structurée en toute saison, mais avec une structure fragile;

$-g_{4}$ ou indice d'une contrainte faible, avec un enracinement à $60 \mathrm{~cm}$, un horizon de pseudogley ou de gley à $60 \mathrm{~cm}$ et plus, une terre arable structurée en toute saison.

L'indice " $g$ " est placé latéralement dans le sigle, comme les autres contraintes (Tableau 1).

Voici, à titre d'exemple, un sol de sigle l.la (b) / $\mathrm{T}_{3} \mathrm{~K}_{4}$. II comporte une terre arable de texture limoneuse battante; les matériaux pédologiques sous-jacents sont ensuite constitués d'un horizon limono-argileux meuble, colonisé par les racines de 20 à $40 \mathrm{~cm}$, et d'un horizon tassé, induré, de 40 à $60 \mathrm{~cm}$; ce dernier, qui ne renferme que de rares racines, doit être considéré comme l'obstacle de l'enracinement; il repose sur un banc de calcaire dur rencontré à plus de 60 $\mathrm{cm}$ de profondeur.

\section{Principe et modalités du classement des sols agricoles}

\section{Principe}

La méthode vise à classer les sols en fonction de leur aptitude à la mise en culture et à les ranger parmi les 6 classes de valeur suivantes: classe 1: sols d'excellente valeur, classe II: sols de très bonne valeur, classe III: sols de bonne valeur, classe IV: sols de valeur moyenne, classe V: sols de valeur inférieure, classe VI: sols de valeur très inférieure.

Les critères de classement pris en compte se subdivisent en 3 groupes:

- un premier groupe en relation avec les conditions d'emplacement: il s'agit du climat et de la pente;

- un deuxième groupe dépendant de l'organisation des sols et de l'interface sol-roche: ce sont le régime atmosphérique (renouvellement de l'air), étroitement corrélé au régime hydrique (Bruckert, 1986b), la profondeur "rhizofonctionnelle" et la nature de l'obstacle à l'enracinement,

- un troisième groupe en rapport avec la composition et les propriétés intrinsèques des constituants: la texture et la pierrosité.

Chaque critère intervient dans l'ordre indiqué cidessus, le long d'un cheminement dichotomique.

\section{1) Critères liés aux conditions d'emplacement}

Le premier facteur - le climat - détermine l'étendue de la zone d'application (Fig. 1) en limitant le choix des cultures (somme des températures, pluviométrie) et en imposant la profondeur utile nécessaire à la constitution des réserves en eau du sol, compte tenu des déficits hydriques saisonniers.

L'analyse statistique sur 25 ans des données climatiques publiées par les services de la Météorologie nationale porte sur une trentaine de postes pluviométriques et thermiques de la région étudiée. Le climat se rattache au type tempéré froid, partiellement nuancé du type semi-continental, avec une saison hivernale longue (température moyenne annuelle inférieure à $10^{\circ} \mathrm{C}$ ) et une saison estivale réduite qui débute à la mimai et se termine début octobre.

Les précipitations atteignent des valeurs moyennes annuelles comprises entre 800 et $1100 \mathrm{~mm}$ selon un gradient croissant d'ouest en est, soit progressivement de Gray à Belfort, soit rapidement au sud d'une ligne Marnay-Besançon. La pluviométrie se répartit tout au long de l'année, mais ce caractère atlantique fait souvent place au caractère continental du climat marqué par des hivers rigoureux et des été chauds soumis à des déficits hydriques atteignant fréquemment $100 \mathrm{~mm}$.

Dans les terres saines prospectées par les racines sur $60 \mathrm{~cm}$, la réserve utile en eau s'élève à $120 \mathrm{~mm}$ en moyenne. II s'agit surtout de sols limono-argileux à argilo-limoneux. Cette valeur s'apparente à la classe moyenne à forte capacité de stockage pour l'eau définie par le GEPPA (1981). On estime régionalement qu'un déficit hydrique s'observe une année sur deux en juillet et août. Dans les terres saines sur $35 \mathrm{~cm}$, cette réserve tombe à $70 \mathrm{~mm}$ (classe moyenne du GEPPA) et le déficit hydrique se produit 1 année sur 3 en juin, 2 années sur 3 en juillet et août. Le risque devient donc important pour les cultures à cycle végétatif estival comme le maïs. La croissance des plantes et les rendements deviennent encore plus aléatoires quand la profondeur racinaire est limitée à une vingtaine de cm: on entre alors dans la catégorie des terres à très haut risque de déficit hydrique.

Le deuxième critère pris en compte est la pente qui limite l'exploitation mécanisée des sols. On estime que des pentes inférieures à 10\% n'entraînent pas de difficultés pour les travaux mécanisés. En revanche, une pente de 10 à $15 \%$ devient une contrainte sérieuse, et une pente supérieure à $15 \%$ une contrainte absolue. 
Dans ce dernier cas, rare sur les surfaces agricoles, la pente détermine à elle seule le classement de valeur.

\section{2) Critères liés à l'organisation des sols et de l'interface sol-roche}

Ces critères dépendent des caractéristiques inhérentes aux sols et doivent de ce fait être recherchés dans les profils pédologiques eux-mêmes et dans les matériaux géologiques sous-jacents. C'est pourquoi, parvenus à ce stade de la démarche, nous employons le sigle de désignation des sols définis dans le paragraphe «Principe et modalités d'expression du sigle de désignation des sols".

Le régime atmosphérique du sol - convenablement aéré ou au contraire anoxique - constitue le troisième critère de classement. Sa place s'impose immédiatement après la pente parce que l'oxygène est, avec l'eau, le premier besoin physiologique des racines.

La profondeur rhizofonctionnelle (quatrième critère) tient compte des déficits hydriques climatiques susceptibles de se produire entre juin et août et du cycle végétatif des plantes cultivées. Nous avons sélectionné les profondeurs de $35 \mathrm{~cm}$ et de $60 \mathrm{~cm}$ qui permettent d'assurer respectivement l'alimentation en eau des plantes dont le cyle se termine en début d'été et des plantes dont le cycle se prolonge jusqu'en automne. Nous avons aussi retenu une profondeur de $20 \mathrm{~cm}$ parce qu'elle s'étend sur de larges surfaces des paysages karstiques. La prise en compte des seuils à 20 , 35 et $60 \mathrm{~cm}$ est donc régionale. Ces profondeurs rhizofonctionnelles sont évidemment "en phase" avec les profondeurs d'apparition de l'obstacle définies dans le paragraphe «Principe et modalités d'expression du sigle de désignation des sols".

L'obstacle à l'enracinement constitue ensuite le cinquième critère pris en compte. II est soit virtuel comme l'anoxie que nous venons de traiter, soit réel ou physique comme une roche ou un horizon inapte à la pénétration des racines. Dans ce cas, nous distinguons des obstacles durs, meubles secs ou meubles frais. Les premiers rendent impossible ou difficile la pénétration de la tarière. Les seconds correspondent à des limons silicieux tassés (horizon $\mathrm{A}_{2}$ lavé des pseudogleys de placages limoneux) et à des limons, ou sables, ou graviers secs. Les troisièmes sont encore des limons, sables ou graviers, mais alimentés par une nappe phréatique.

L'identification de l'obstacle permet d'une part de savoir si la profondeur d'enracinement peut être augmentée et comment on peut y parvenir et, d'autre part, d'expliquer les principaux comportements de la terre arable et des plantes face aux influences saisonnières et aux travaux culturaux.

\section{3) Critères liés aux constituants}

Le comportement et les propriétés des constituants dépendent de critères intrinsèques (taille, propriétés physico-chimiques, composition chimique, altérabilité...) et de critères de fonctionnement du sol. La texture ne prend toute sa signification que compte tenu du régime hydrique, de l'environnement ionique, de la qualité et du taux de matière organique. On observe couramment des terres de même texture qui se comportent de façons très différentes. C'est la raison pour laquelle nous plaçons en dernière position les critères liés aux constituants.

En conditions aérées et d'environnement ionique favorable à la floculation des colloïdes organo-miné- raux, c'est en effet la texture qui détermine en grande partie la structure et le comportement de la terre. On observe que les terres limono-argileuses et argilolimoneuses saines se caractérisent par une meilleure agrégation, à la fois stable et fine, parce que la teneur en argile est suffisante, sans être excessive. Les terres trop limoneuses ou trop sableuses ont par contre une stabilité structurale médiocre, liée à un manque de capacité d'échange cationique: elle sont battantes et sensibles à l'érosion. Les terres trop argileuses se structurent, mais sont saisonnièrement trop plastiques ou trop cohérentes et accroissent les diffjcultés de travail du sol.

En sol anoxique, ce n'est pas la texture qui détermine la structure et le comportement de la terre, mais le régime hydrique. En période de saturation par l'eau, le milieu privé d'oxygène devient réducteur. Les oxydes de fer qui adhèrent en revêtement sur les argiles (Rich et Thomas, 1960) sont déstabilisés. Leur action bénéfique sur la structure cesse, et les particules se dissocient les unes des autres. Les effets de l'anoxie sont observés quelle que soit la texture (Bruckert, 1986 b).

Parmi les contraintes liées aux constituants, la pierrosité devient une gêne pour les outils aratoires quand elle atteint un seuil élevé, d'environ $50 \%$ en volume. C'est ce taux, adopté également par le GEPPA (1981), que nous avons pris en considération.

\section{Clé dichotomique de classement des sols agri- coles}

La clé dichotomique introduit successivement les critères par ordre d'importance décroissante: climat, pente, régime atmosphérique, profondeur rhizofonctionnelle, obstacle à l'enracinement, texture et pierrosité. Elle oppose à chaque niveau une propriété favorable à une contrainte. Le choix dichotomique conduit soit au niveau suivant, soit au classement (Tableau II).

La Figure 2 en donne un extrait synoptique se rapportant aux sols de pente inférieure à $10 \%$. On y suit clairement les itinéraires de classement. Nous en avons matérialisé deux correspondant aux sols de sigles $\mathrm{al} / \mathrm{K}_{3}$ (sol brun) et la $\left(\mathrm{g}_{3}\right) / \mathrm{T}_{3} \mathrm{y}_{2}$ (sol brun à pseudogley):

- le premier aboutit à la classe II en empruntant le chemin "sols aérés - profondeur rhizofonctionnelle inférieure à $60 \mathrm{~cm}(35$ à $60 \mathrm{~cm})$ - obstacle dur - texture équilibrée - pierrosité nulle";

- le second conduit à la classe IV en parcourant le tracé "sols anoxiques - taches d'oxydo-réduction audessus de $60 \mathrm{~cm}$, à partir de $20 \mathrm{~cm}$ - intensité de l'anoxie".

Au total, le classement des sols de pente inférieure à $10 \%$ qui couvrent la majeure partie des surfaces agricoles offre 45 possibilités. Les fréquences des classes se distribuent sur une courbe normale unimodale (Tableau III). L'écart observé entre la médiane et la moyenne provient des sols anoxiques dont le classement débute à la classe III. Nous avons jugé en effet que l'anoxie liée au régime hydrique défectueux constitue un facteur limitant sévère pour la facilité de mise en culture (Jones, 1979). Néanmoins, pour en nuancer la pénalisation nous avons adopté une démarche en deux temps qui introduit successivement la profondeur d'apparition des taches d'oxydo-réduction, puis l'intensité de l'anoxie. On retrouve ce souci chez Arrouays (1987). 
Tableau II. Extrait de la "Clé dichotomique de classification des sols agricoles" (Bruckert, 1987).

$1\{$ Per

Pente supérieure à $15 \%$

Pente inférieure à $15 \%$

Classe VI

$2\{$ Pente de 10 à $15 \%$

Pente inférieure à $10 \%$.

3.

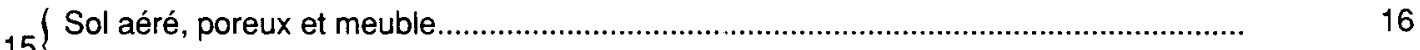

15\{

Sol mal aéré ou anoxique...

16 Profondeur "rhizofonctionnelle" supérieure à $60 \mathrm{~cm}$

Profondeur "rhizofonctionnelle" inférieure ou égale à $60 \mathrm{~cm}$.

(Textures "équilibrées" 1 , limono-argileuses, argilo-limoneuses ou limoneuses calcaires

Sigles: la ou (al ou l* / obstacle 4) ou la' ou al' ou l'-

Classe I

17

Textures déséquilibrées, argileuses ou limoneuses ou sableuses

Sigles: (al / obstacle 4) tou al't ou (l ou sl / obstacle 4) b ou l'b ou sl'b ou s'b-

Classe II

18 Profondeur "rhizofonctionnelle" de 35 à $60 \mathrm{~cm}$.

$19 \ldots$

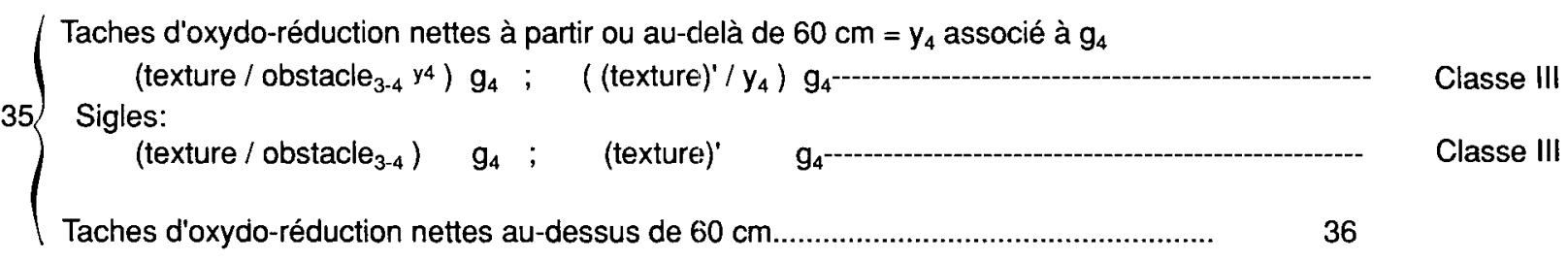

$36 \ldots$

1 "équilibrées" au sens de favorable à la structuration grumeleuse.

\section{Discussion et conclusion}

La méthode de désignation des sols présentée dans ce texte permet de transcrire dans un langage objectif et informatisable les caractéristiques concernant les constituants, les propriétés essentielles et les contraintes des sols agricoles.

Le sigle proposé est un outil de connaissance qui peut s'employer indépendamment de la clé de classement. Appliqué dans le contexte régional des plateaux francs-comtois de basse altitude, il conduit à définir un nombre limité de sols types désignés dans le Tableau IV. Les sols se

Tableau III. Fréquence des classes de valeur portant sur l'examen des sols de pente inférieure à $10 \%$.

$\begin{array}{lllllll}\text { Classes } & \text { I } & \text { II } & \text { III } & \text { IV } & \text { V } & \text { VI } \\ \text { Fréquences } & 2 / 45 & 6 / 45 & 12 / 45 & 13 / 45 & 9 / 45 & 3 / 4.5\end{array}$

subdivisent en plusieurs séries qui diffèrent fondamentalement entre elles par leurs matériaux constitutifs et leur environnement géologique.

La clé dichotomique de classement, quant à elle, met en jeu l'interaction climat-sol dans l'utilisation polyvalente des terres agricoles. Elle place à un niveau élevé l'aération des horizons sous-jacents à la terre arable, en raison du rôle fondamental de cette caractéristique physique dans le développement des systèmes racinaires. Dans notre démarche, le régime hydrique est, par voie de conséquence, considéré comme un facteur limitant essentiel. Quand il est défectueux, il contrecarre l'optimisation de la fertilisation et accroit les difficultés d'exécution des travaux culturaux. Nous rejoignons en cela Jones (1979) et Haans et al. (1984) qui soulignent également l'importance des caractères physiques et la nécessité d'une meilleure quantification des régimes hydriques (Thomasson et Jones, 1988).

Le principe de la méthode proposée peut être facilement appliqué hors du contexte régional: il 
Tableau IV. Désignation des sols des plateaux calcaires et marno-calcaires francs-comtois: les sols sont rangés selon leur caractère aéré ou anoxique et leur appartenance à une série définie par les matériaux constitutifs; la correspondance entre les sigles et les types génétiques de la classification française CPCS (1967) est donnée à hauteur de la série.

Séries de sols définies par les matériaux constitutifs
Plateaux calcaires et marno-calcaires diaclasés
sols à régime aéré

Profonds et moyennement profonds Superficiels
Série de placage limono-argileux ou limoneux

\section{Série de colluvium} limono-argileux ou limoneux

\section{Série d'altérite} décarbonatée argileuse et limoneuse

Série d'altérite carbonatée argileuse et limoneuse

$\frac{\text { la.al }}{\mathrm{T} \text { ou } \mathrm{TO}_{3-4} \mathrm{~K}_{34}}$ ou $\frac{\text { l.la }}{\mathrm{T}_{\text {ou } \mathrm{TO}_{3-4} \mathrm{~K}_{3-4}}} \frac{\text { la ou al }}{\mathrm{T} \text { ou } \mathrm{TO}_{2} \mathrm{~K}_{3-4}}$ ou $\frac{1}{\mathrm{~T} \text { ou } \mathrm{TO}_{2} \mathrm{~K}_{3-4}}$ b

Brun lessivé de placage

la ou 1

Brun lessivé tronqué de placage la ou 1

la' ou l'b

Colluvial brunifié de colluvium

la ou I

$$
\frac{\text { al ou la }}{\mathrm{K}, \mathrm{J}, \mathrm{O} \text { ou } \mathrm{M}_{3-4}}
$$

Brun et brun calcique d'altérite décarbonatée al ou la

$$
\frac{\mathrm{al}^{*} \text { ou } \mathrm{la}^{*}}{\mathrm{~J} \text { ou } \mathrm{M}_{3-4}}
$$

Brun calcaire d'altérite carbonatée al* ou la*

\section{$\frac{\text { al ou la }}{\mathrm{K}, \mathrm{J}, \mathrm{O} \text { ou } \mathrm{M}_{2}}$}

Brun à pellicule calcaire et brun calcique superficiel d'altérite décarbonatée al ou la

$\frac{\text { al }^{*} \text { ou la* }}{\mathrm{J} \text { ou } \mathrm{M}_{2}}$

Brun calcaire superficiel d'altérite carbonatée $\mathrm{al}^{*}$ ou la*
Séries de sols définies par les matériaux constitutifs
Plateaux calcaires et marno-calcaires

Peu diaclasés

Compacts

Sols à régime anoxique

Modéré $\left(g^{4}\right)$ à moyen $\left(g^{3}\right)$

Fort $\left(g^{2}\right)$

$\frac{\text { la }}{\mathrm{Ty}_{2-4}} \mathrm{~g}_{3-4}$ ou $\frac{1}{\mathrm{Ty}_{2-4}} \mathrm{bg}_{3-4}$

Brun lessivé à pseudogley de placage la ou 1

Série de colluvium limono-argileux ou limoneux

\section{Série d'altérite} décarbonatée argileuse et limoneuse

Série d'altérite carbonatée argileuse et limoneuse $\frac{l^{\prime}}{y_{2-3}} g_{3-4}$ ou $\frac{l^{\prime}}{y_{2-3}} b_{3-4}$

Colluvial à pseudogley de colluvium

$$
\frac{\text { al ou la }}{\mathrm{O} \text { ou } \mathrm{My}_{2-4}} \mathrm{~g}_{3-4}
$$

Brun à pseudogley d'altérite décarbonatée al ou la

$\frac{\mathrm{al}^{*} \text { ou } \mathrm{la}^{*}}{\mathrm{~J} \text { ou } \mathrm{M}_{3-4}} \mathrm{~g}_{3-4}$

Brun calcaire à pseudogley d'altérite carbonatée $\mathrm{al}^{*}$ ou la* $\frac{\text { la }}{\mathrm{Ty}_{1-2}} \mathrm{~g}_{2}$ ou $\frac{1}{\mathrm{Ty}_{1-2}} \mathrm{bg}_{2}$

Pseudogley de placage la ou 1

$\frac{\mathrm{la}^{\prime}}{\mathrm{y}_{1-2}} \mathrm{~g}_{2}$ ou $\frac{\mathrm{l}^{\prime}}{\mathrm{y}_{\mathrm{i}-2}} \mathrm{bg}_{2}$

Pseudogley de colluvium la ou 1

$\frac{\text { al ou la }}{\mathrm{O} \text { ou } \mathrm{My}_{1-2}} \mathrm{~g}_{2}$ ou $\frac{1}{\mathrm{O} \text { ou } \mathrm{M}_{\mathrm{y}} \mathrm{y}_{1}}$

Pseudogley d'altérite décarbonatée al, la ou I 


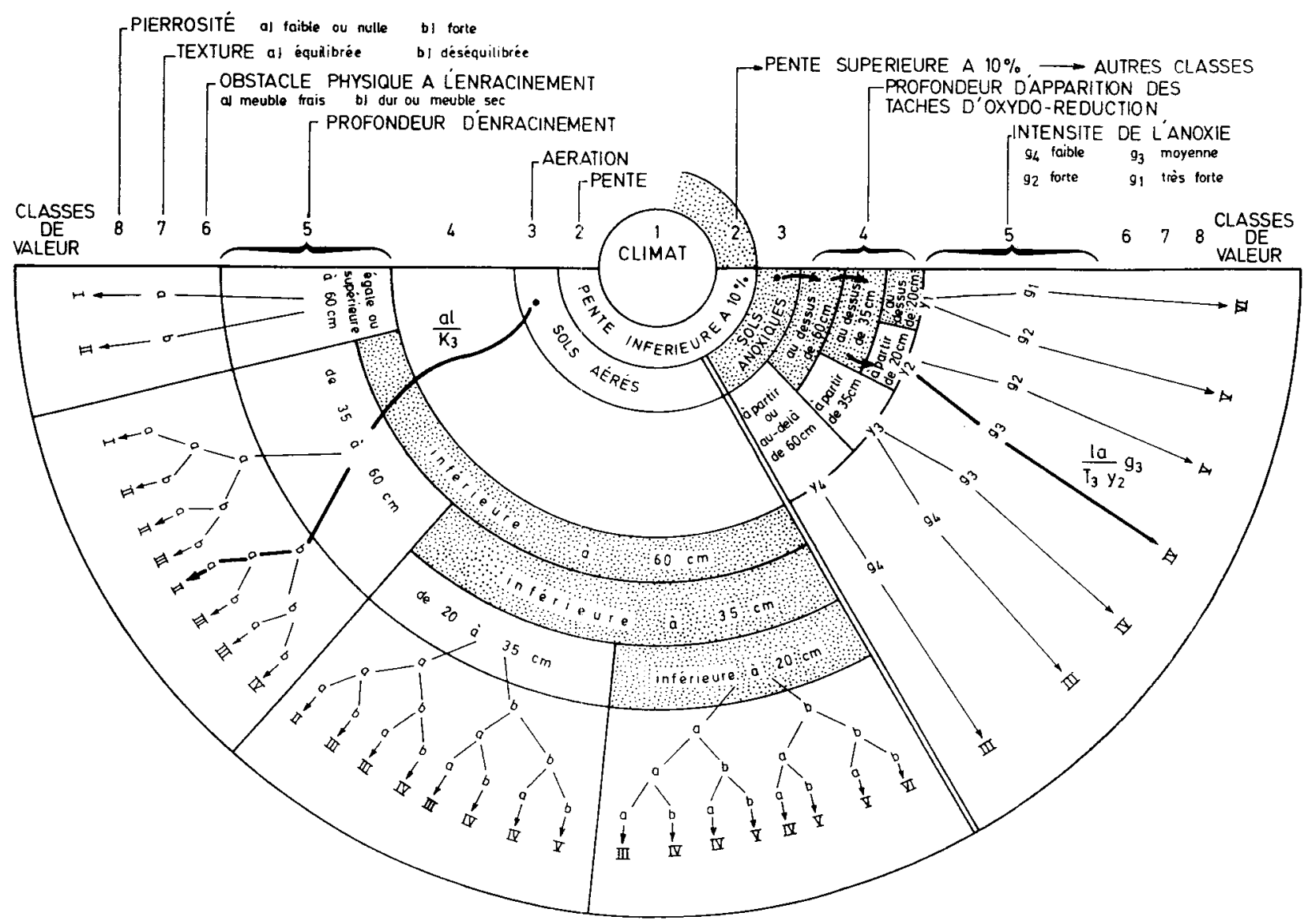

Fig. 2. Distribution des classes de valeur en fonction de l'entrée successive de facteurs hiérarchisés ( 8 en tout) et du chemin dichotomique suivi: cette distribution ne concerne que les sols dont la pente est inférieure à $10 \%$.

suffit pour cela d'adapter la clé et de prendre en compte les critères spécifiques de la nouvelle zone concernée. C'est un outil de synthèse performant en cartographie des sols à but finalisé et dans le choix des aménagements du domaine rural.

La combinaison de ces deux approches permet de dresser des cartes à grande échelle (Bruckert, 1986b), qui fournissent de façon analytique les propriétés et les contraintes des sols et qui visualisent de façon synthétique les classes de valeur pédologique et agronomique des terres.

\section{Remerciements}

L'auteur remercie MM. C. de Kimpe, J. Dejou et M. Jamagne pour les remarques constructives et pertinentes apportées à l'amélioration du texte.

\section{Références}

Arrouays D. (1987) Cartographie des sols et comportements agronomiques Sci. Sol 25 (1) 43-58

Begon J.C., Mori A. \& Hardy R. (1978) Un système de classement des terres suivant leur aptitude à la pro- duction agricole. Son application à une région de culture intensive dans le Bassin parisien. C.R. Séances Acad. Agric. Fr. 1274-1285

Bibby J.S. \& Mackney D. (1969) Land use capability classification. Soil Surv. Tech. Monogr. 1, 1-12

Boiffin J., Meynard J.M., Sebillotte M. \& Caneill J. (1982) Elaboration du rendement et fertilisation azotée du blé d'hiver en Champagne crayeuse. III. Influence des variations pédologiques; conséquences pour la conduite de la culture. Agronomie 2, 5, 417-428

Bruckert S. (1986a) Méthode de désignation des sols appliquée à la connaissance des terres agricoles des plateaux francs-comtois de basse altitude. Ann. Sci. Univ. Besançon Biol. Vég. 4, 6, 31-36

Bruckert S. (1986b) Carte de la Valeur Pédologique et Agronomique des Terres des Hauts du Val-de-Saône. Notice générale, Université de Besançon

Bruckert S. (1987) Clé dichotomique de classement des sols agricoles francs-comptois situés en climat tempéré semi-continental. Ann. Sci. Univ. Besançon Biol. Vég. 4, 7, 1-15

Commission canadienne de pédologie. 1978. Comité de Classification des Sols. Le Système Canadien de la Classification des Sols. Ministère de l'Agriculture du Canada, Ottawa, publication 1646

Callot G. (1984) Structure pédologique et fonctionnement du sol en relation avec la production végétale. Analyse au niveau parcellaire. Bull Assoc. Fr. Etude Sol 2, 167-181 
Callot G., Chamayou H., Maertens C. \& Salsac L. (1983) Mieux Comprendre les Interactions SolRacines. Incidence sur la Nutrition Minérale. INRA, Paris

Duclos G. (1971) Appréciation de l'aptitude à la mise en valeur des sols de Provence. Bull. Assoc. Fr. Etude Sol 6, 33-46

Geppa (1981) (Groupe d'Etude des Problèmes de Pédologie Appliquée) Synthèse des Travaux de la Commission de Cartographie, pp. 1-20

Grable A.R. \& Siemer E.G. (1968) Effects of bulk density, aggregate size, and soil water suction on oxygen diffusion, redox potentials, and elongation of Corn roots. Soil Sci. Soc. Am. Proc. 32, 180-186

Guide pour l'estimation de domaine et de bien-fonds agricoles (1979) Office fédéral du matériel, Berne

Haans J.C.F.M., Steur G.G.L. \& Heide G. (1984) Progrès dans l'évolution des terres. Compte-rendu d'un séminaire sur la cartographie des sols et l'évaluation des terres, Wageningen (Hollande), 26-29 septembre, Rotterdam, A.A. Balkema

Hallaire V. (1981) L'évaluation des sols de prairie permanente. Thèse de doctorat d'ingénieur. Université de Rennes

Harrod T.R. (1979) Soil suitability for grassland. Soil Surv. Applic. Soil. Surv. Technic. Monogr. 13, 51-70

Henin S. (1960) Le Profil Cultural. Principes de Physique du Sol. Edition des Ingénieurs Agricoles, Paris

Jamagne M., Maucorps J., Bliet L., Bouttemy R. \& Rivière J.-M. (1966) Principes de classification régio- nale des sols. Sci. Sol 2, 41-64

Jones R.J.A. (1975) Soils in Staffordshire II: Sheet SJ82 (Eccleshall). Soil Surv. Rec. 31

Jones R.J.A. (1979) Soils of the Western Midlands grouped according to Ease of Cultivation. Soil Surv. Applic. Soil Surv. Technic. Monogr. 13, 24-42

Lajoie P.G. (1975) Les terres agricoles du Québec méridional, distribution, étendue et qualité. Agriculture Canada, publication 1556, 62 p. + 1 carte

Mathieu D. (1976) Atlas de Franche-Comté. 6. Institut de géographie, Université de Besançon

Mondon G. (1978) Pédologie de classement des terres pour le remembrement. INRA, Paris, Grignon

Mori A., Begon J.C., Duclos G., Studer R., 1983. Progress in land evaluation. Proc. Seminar on Soil Surv. and Land Evaluation. Wageningen, Hollande, 26-29 sept., pp. 43-55

Rich C.I. \& Thomas G.W. (1960) The clay fraction of soils. Adv. Agron. 12, 1-39

Russel M.B. (1949) Methods for measuring soil structure and aeration. Soil Sci. 68, 25-35

Sebillotte M., Boiffin J., Caneill J. \& Meynard J.M. (1978) Sécheresse et fertilisation azotée du blé d'hiver. Essai d'analyse du champ par l'étude des composants de rendement. Sci. Sol 3, 197-214

Thomasson A.J. (1971) Soils of the Melton Nowbray district. Mem. Soil surv. Gt. Br.

Thomasson A.J. \& Jones R.J.A. (1988) Land evaluation at reginal scale. Symposium on land qualities in space and times. Wageningen, The Netherlands, August 22-26. 